\title{
DATABASES
}

\section{The Italian XLMR Bank: A Clinical and Molecular Database}

\author{
C. Pescucci, R. Caselli, F. Mari, C. Speciale, F. Ariani, M. Bruttini, K. Sampieri, M.A. Mencarelli, E. Scala, \\ I. Longo, R. Artuso, A. Renieri, ${ }^{*}$ and I. Meloni, and the Members of the XLMR Italian Network \\ Medical Genetics, Department of Molecular Biology, University of Siena, Siena, Italy
}

Communicated by Jamie Cutticchia

\begin{abstract}
Mental retardation (MR) is a nonprogressive condition characterized by a significant impairment of intellectual capabilities with deficit of cognitive and adaptive functioning and onset before 18 years. Mental retardation occurs in about 2 to $3 \%$ of the general population and it is estimated that 25 to $35 \%$ of the cases may be due to genetic causes. Among these "genetic" MR, 25 to $30 \%$ are probably due to mutations in a gene on the X chromosome (X-linked mental retardation, XLMR). Given the genetic heterogeneity of XLMR, the availability of a considerable number of patients with accurate phenotypic classification is a crucial factor for research. The X-linked Mental Retardation Italian Network, which has been active since 2003, has collected detailed clinical information and biological samples from a vast number of MR patients. Collected samples and clinical information are inserted within the XLMR bank, a comprehensive molecular and clinical web-based database available at the address http://xlmr.unisi.it. The database is organized in three distinct parts. Part I and II contain several electronic schedules to register information on the family, the phenotypic description, the photographs, and a $20 \mathrm{sec}$ movie of the patient. Part III allows the registration of molecular analyses performed on each case; samples and clinical data are usable via password-restricted access. Clinical and molecular centers interested in joining the network may request a password by simply contacting the Medical Genetics of the University of Siena. The XLMR bank is an innovative biological database that allows the collection of molecular and clinical data, combines descriptive and iconographic resources, and represents a fundamental tool for researchers in the field of mental retardation. Hum Mutat 28(1), 13-18, $2007 . \quad$ (c) 2006 Wiley-Liss, Inc.
\end{abstract}

KEY WORDS: XLMR; X-linked mental retardation; molecular database; clinical database; XLMR Italian Network

\section{INTRODUCTION}

Mental retardation (MR) is a nonprogressive condition characterized by a significant impairment of intellectual capabilities with deficit of cognitive and adaptive functioning and onset before 18 years [Chelly and Mandel, 2001]. General intellectual functioning is defined by the intelligence quotient (IQ) and adaptive functioning refers to how individuals deal with common life demands. Mental retardation occurs in about 2 to $3 \%$ of the general population [Gecz, 2004] and represents an important socioeconomic and medical issue, given that MR patients need a continuous support from families and healthcare operators. It is estimated that a percentage of MR cases between 25\% and 35\% may be due to genetic causes. On the basis of the IQ value, mental retardation may be classified in four categories of severity: mild (IQ 50-70), moderate (IQ 35-50), severe (IQ 20-35), and profound $(\mathrm{IQ}<20)$. Moreover, MR may be present in association with other clinical manifestations (syndromic MR) or may be isolated (nonsyndromic MR) [Mulley et al., 1992]. Males are more frequently affected than females, with an excess of about $30 \%$ of male cases explained by the presence of several causative genes on the X chromosome responsible for the X-linked cases of MR (XLMR) [McLaren and Bryson, 1987; Baird and Sadovnick, 1985; Neri and Chiurazzi, 1999]. At present, 27 genes for nonsyndromic and 45 genes for syndromic XLMR are known [Renieri et al., 2005; Bauters et al., 2005]. Some genes are responsible for both syndromic and non-syndromic XLMR and most of them are responsible for a very low percentage of cases, comprising between 0.1 and $1 \%$.

The large number of known XLMR genes and the fact that no major genes exist makes it difficult to offer a molecular diagnosis to MR patients. Given the genetic heterogeneity of XLMR, the availability of a considerable number of patients with accurate phenotypic classification is a crucial factor for research. The X-linked Mental Retardation Italian Network includes 12 laboratories and 27 clinical centers and has been active since 2003, collecting MR patients with detailed clinical information according to common criteria and analyzing known XLMR genes.

The Supplementary Material referred to in this article can be accessed at http://www.interscience.wiley.com/jpages/1059-7794/ suppmat.

Received 21 April 2006; accepted revised manuscript 18 July 2006.

*Correspondence to: Alessandra Renieri, MD, PhD, Medical Genetics, Molecular Biology Department, University of Siena, V.Le Bracci, 53100 Siena, Italy. E-mail: renieri@unisi.it

Grant sponsor: Pierfranco e Luisa Mariani Foundation; Grant sponsor:Telethon Foundation; Grant numbers: GTF02006, GTF05005.

The members of the XLMR Italian Network are listed in Appendix A. DOI 10.1002/humu.20411

Published online 18 September 2006 in Wiley InterScience (www. interscience.wiley.com) 
It collects both sporadic and familial mentally retarded male patients negative for Fragile X Mental Retardation Syndrome (FRAXA), chromosomal and subtelomeric rearrangements. Biological samples are conserved in a biobank located at the Medical Genetics laboratory of the University of Siena and at the Genetic Institute of the Università Cattolica del Sacro Cuore in Rome. Collected samples and clinical information are inserted in a webbased database available at the address http://xlmr.unisi.it through a password-restricted access. All centers interested in joining the Network can contact the Medical Genetics of the University of Siena.

We describe here the "XLMR bank," a comprehensive webbased database that includes accurate clinical and molecular data on the MR patients present in the biobank. Samples and clinical data are available upon request for specific studies. The XLMR bank represents an important tool for researchers involved in the discovery of the molecular genetic bases of autosomal and X-linked $\mathrm{MR}$ and the clarification of the pathogenic processes that underlie the disease.

\section{THE XLMR BANK}

The XLMR bank is available at the web address http:// xlmr.unisi.it and it is maintained and updated on a server at the University of Siena. The database was created using Access software (Microsoft Office, Microsoft; www.microsoft.com) for data management. The website is written in Virtual Basic script
(VBScript) and it takes advantage of a Microsoft Internet Information Server (IIS) with Active Server Pages (ASP) technology.

\section{Database Structure}

The home page of the XLMR bank (http://xlmr.unisi.it/homepage.asp) contains general information about the project. In particular, it explains the aims and the overall organization of the project (clinical centers and laboratories involved, patients enrolled, and genes analyzed). Using the "Search" option, external users can visualize the complete list of patients and pedigree and provisional or definitive diagnosis for each patient (Fig. 1).

The overall design of the XLMR bank database includes three distinct parts with password-restricted access (Supplementary Figure S1; available online at http:/www.interscience.wiley.com/ jpages/1059-7794/suppmat). Each part is organized in several electronic schedules, to collect detailed clinical and molecular data. Part I includes forms filled by the clinical center that has visited the patient and collected the biological samples (Fig. 2). The forms are divided in the following specific sections: 1 ) private data; 2) family history (pedigree, clinical data, and relatives' photographs); 3) anamnestic form (clinical history of the patient, IQ value, adaptive functioning evaluation and behavioral tests, laboratory results for standard cytogenetic analysis, investigation of subtelomeric rearrangements, and fragile-X test); 4) clinical data of the patient (clinical features, morphologic examination, neurological data, JPEG format photographs, and $20 \mathrm{sec}$ movie

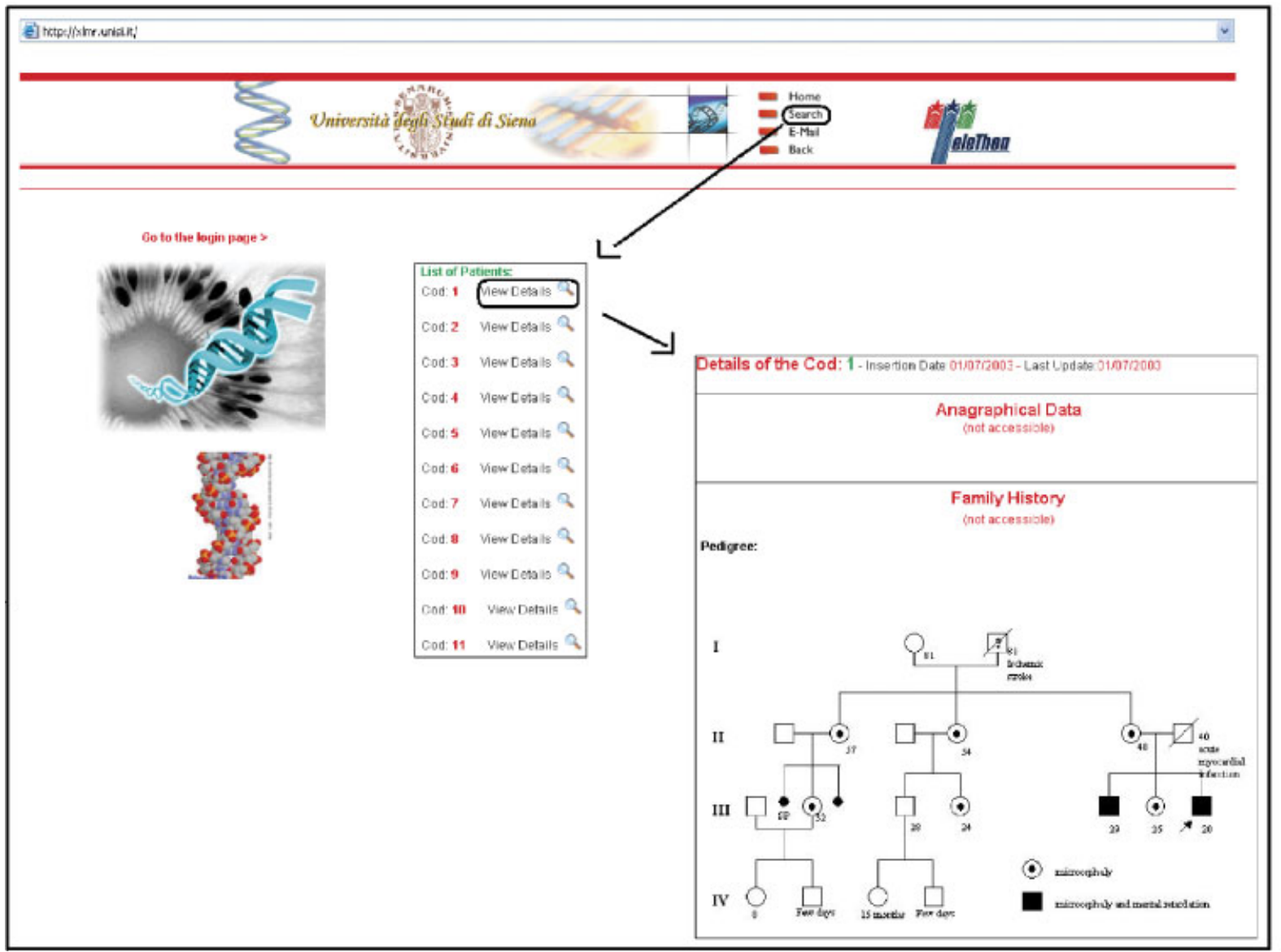

FIGURE 1. Home page of the XLMR bank (http://xlmr.unisi.it). Using the "Search" option available at the home page, users can visualize the complete list of patients recorded in the bank and consult their pedigree. This set of information is available also for "external users" without password access. [Color figure can be viewed in the online issue, which is available at www.interscience. wiley.com.] 


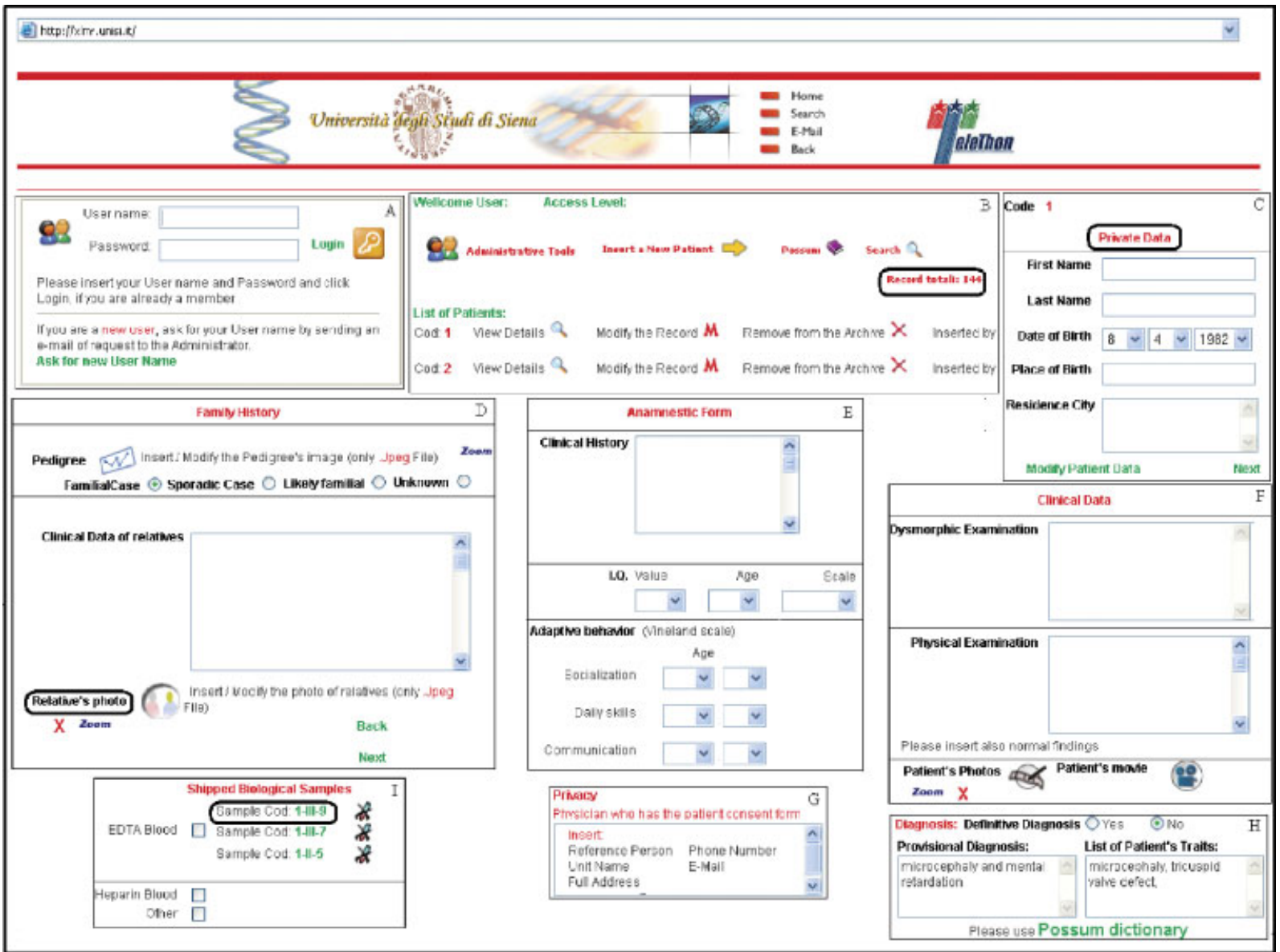

FIGURE 2. Part I of the XLMR bank database. A: Access to this section is password-restricted. B-I: The different schedules included in this section and filled in by the clinical center that has visited the patient. [Color figure can be viewed in the online issue, which is available at www.interscience.wiley.com.]

whenever relevant); 5) privacy (name and address of the physician that retains the informed consent); 6) diagnosis (provisional diagnostic hypothesis); 7) trait description, using conventional dysmorphology dictionary (from London Dysmorphology[LDDB] and Possum databases); 8) shipped biological samples (collected samples and biobank address). To guarantee confidentiality, each sample in the database is univocally identified by a code of three numbers separated by an hyphen: a progressive number generated by the system that characterizes the family and is the same for each family member and two numbers indicating pedigree position of the subject (Fig. 2I).

Part II is completed by biobank curators and it includes information on the source and the type of stored biological samples (Fig. 3). Currently, the following biological samples are stored: DNA, plasma, and lymphocytes and lymphoblastoid cell lines in dimethyl sulfoxide (DMSO) medium.

Part III includes information on laboratory tests performed on each sample (Fig. 4). This part is completed by the laboratories that join the Network and execute the molecular analyses. The laboratory tests currently performed are listed in Table 1 .

\section{Database Accessibility and Clinical and Molecular Data Management}

The database is organized on five levels of admittance, a public level and four different "participant" levels, accessible through a password only to centers belonging to the "X-linked Mental Retardation Italian Network" (Supplementary Figure S1). The first level is freely available to external users going to the "Search" option. External users can freely visualize the pedigree of each case. Specific information is accessible only for participants through a "Log in" page, which includes detailed clinical data, location, and preservation of biological samples (DNA, lymphoblastoid cell lines, plasma, urine samples, etc.) and molecular data. A second level password is assigned to clinical centers that visit the patient and decide to insert the case into the XLMR bank. Clinical centers collect biological samples and send them to the laboratories qualified for storage. A laboratory qualified for storage has to guarantee the establishment and maintenance of lymphoblastoid cell lines. Using the second level password, clinical centers can visualize and modify clinical data limited to patients they have inserted and they can consult but not modify their molecular data. A third level password allows to clinical centers to visualize clinical and molecular data of all patients in the bank. Laboratories that perform molecular analyses have a fourth level password that allows them to complete Part III of the database. Each laboratory can visualize molecular information inserted by other laboratories. The fifth level password is the administrator level. Only bank curators have this access, which allows users to visualize and modify all the information maintained in the database, including anagraphical data.

\section{Security and Quality Assurance}

The database has been constructed in compliance with the guidelines of the Italian Society of Human Genetics and Telethon 


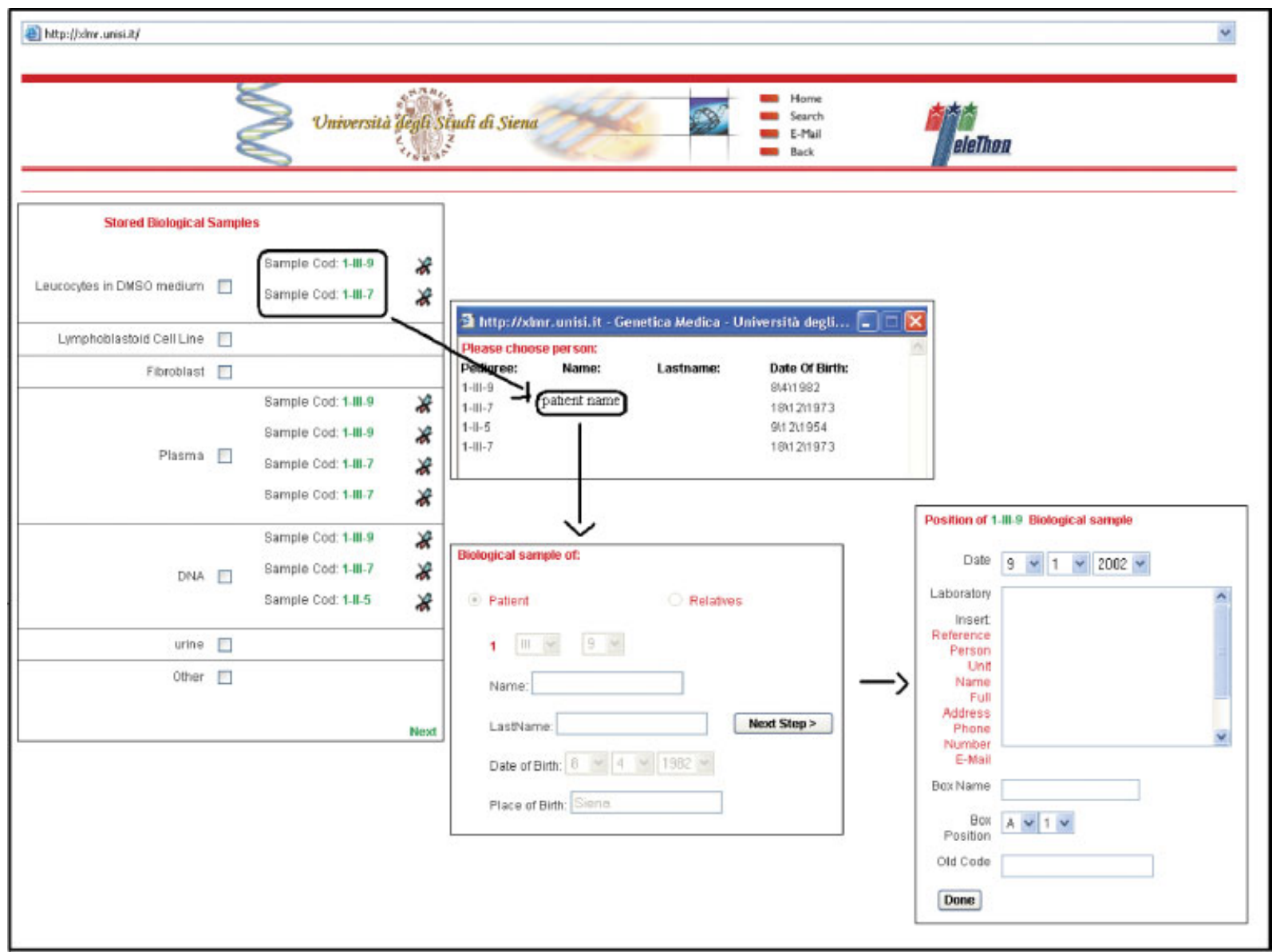

FIGURE 3. Part II of the XLMR bank database. This section of the database includes information on the source and the type of stored biological samples. It is possible to add new samples simply by clicking on the check-box corresponding to the correct sample type stored (i.e., DNA sample). Note that the code is generated by the system when a new patient is registered in the bank. The same code is attributed to each sample obtained from that patient. [Color figure can be viewed in the online issue, which is available at www. interscience.wiley.com.]

Foundation for biobanking [Dagna Bricarelli et al., 2003]. The database design assures patients' anonymity, privacy and confidentiality, according to international criteria [Godard et al., 2003]. Moreover, the informed consent has been written explicitly stating all aspects of stored samples and personal data management [Godard et al., 2003].

The XLMR bank is available to all users who contact bank curators and fill in and sign a specific form asking to join the Network on X-Linked Mental Retardation and to receive a new password. The Network members agree to follow the rules of the bank and commit themselves not to distribute the password and the information maintained in the database. Anonymity of the samples is assured in each step of the research. Only the bank curator has access to the whole information content of the database, including personal data. Neither other members of the Network nor external investigators wishing to take advantage from stored samples can access patients' personal data (anagraphical data, photographs, movies, etc.) and consequently they are not able to identify individuals. Only clinicians with a second level password can visualize personal data limited to patients they enrolled in the database.

Both genomic DNA and lymphoblastoid cell lines (or primary lymphocytes for future cell line establishment) are stored for all probands and for available relatives. DNA aliquots for standard analyses by different network laboratories are prepared immediately upon DNA extraction. Subsequently, two aliquots of at least
$400 \mu \mathrm{g}$ each are prepared and they are stored in $-20^{\circ} \mathrm{C}$ freezers for long-term conservation. Regarding cell cultures, six distinct aliquots for each lymphoblastoid cell line are stored (four aliquots in the $-135^{\circ} \mathrm{C}$ freezer and two in the liquid nitrogen Dewar). Cells are frozen at passage 2 in $1.5-2 \times 10^{7}$ cells $/ \mathrm{ml}$ aliquots. To prevent the accidental damage of samples, storage of biological materials is organized in different $-20^{\circ} \mathrm{C}$ or $-135^{\circ} \mathrm{C}$ freezers.

\section{Database Functions}

Users that visit the online database can search for a patient by simply looking through the list of registered cases. Alternatively, the database may be explored using the "Search" option available after login. The search page allows to browse the database selecting for any field the user wants to search for (Supplementary Figure S2). It is possible to search by a single field or to combine two or more fields. As a result of the search, the user will visualize the list of patients that present the selected feature(s). Information on the selected patients will be available according to the level of the login password.

\section{DISCUSSION}

The primary focus of the XLMR bank is to collect a wide number of both syndromic (unknown syndromes) and nonsyndromic mental retardation cases. Familial cases with an X-linked pattern of transmission are preferred. However, the bank collects 


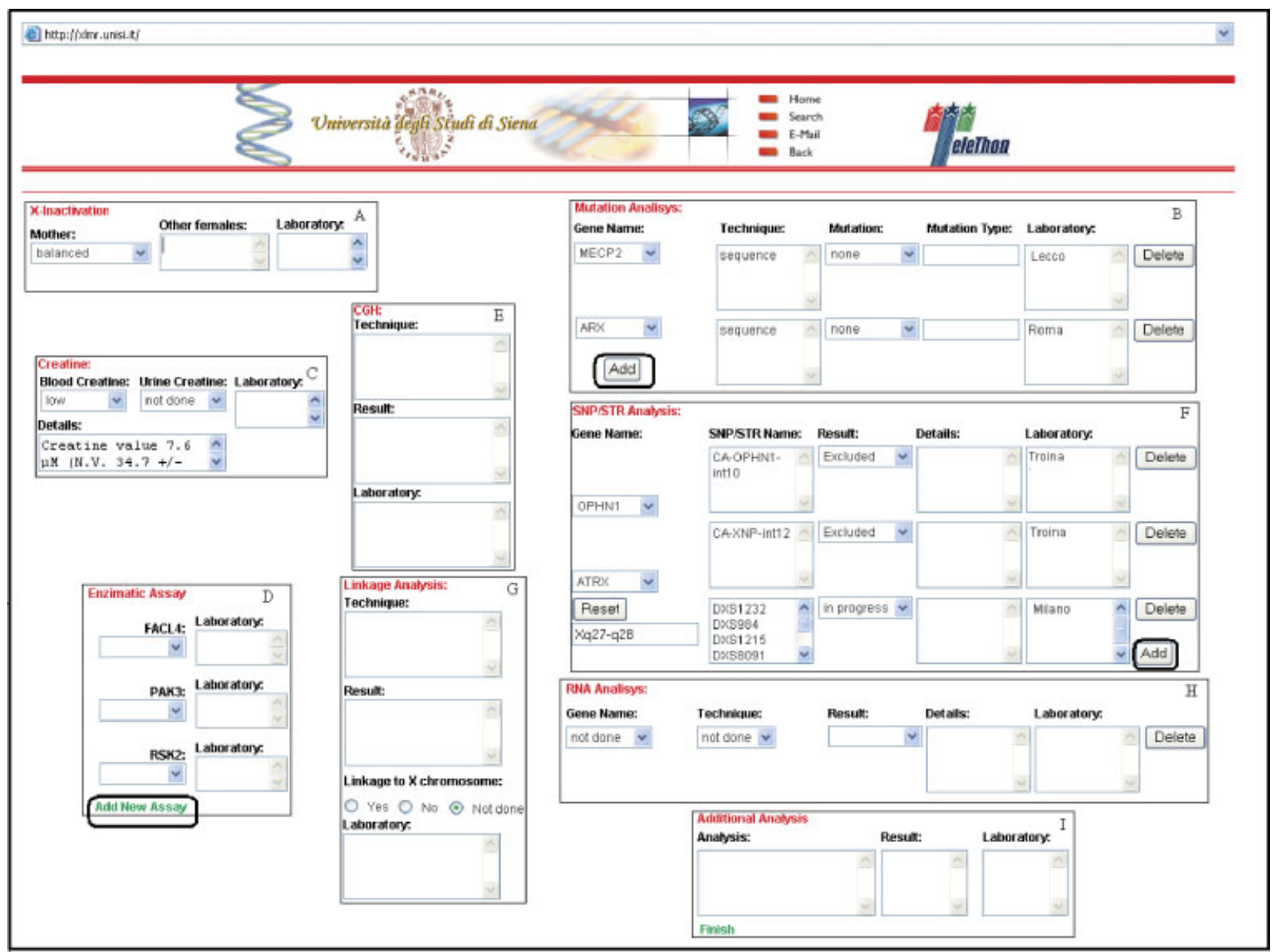

FIGURE 4. Part III of the XLMR bank database. Each panel of this section includes information on the laboratory tests performed on collected samples. Note that different tests may be added or deleted on the basis of the ongoing work. [Color figure can be viewed in the online issue, which is available at www.interscience.wiley.com.]

TABLE 1. Laboratory Tests Currently Executed by the XLMR Italian Network Members

\begin{tabular}{|c|c|c|}
\hline Mutation analysis & Gene name & Laboratory \\
\hline & MECP2 & Lecco \\
\hline & OPHN1 & Lecco \\
\hline & FMR2 & Padova \\
\hline & NLGN3/NLGN4 & Pavia \\
\hline & $D C X$ & Pisa \\
\hline & $A R X$ & Roma \\
\hline & IL1RAPL1 & Roma \\
\hline & AGTR2 & Trieste \\
\hline & ATRX & Troina \\
\hline & PQBP1 & Troina \\
\hline & RSK2 & Troina \\
\hline \multirow{4}{*}{$\begin{array}{l}\text { Enzymatic/biochemical } \\
\text { assays }\end{array}$} & Assay & Laboratory \\
\hline & $\begin{array}{l}\text { Blood creatine } \\
\text { dosage }\end{array}$ & $\begin{array}{l}\text { Firenze, } \\
\text { Genova }\end{array}$ \\
\hline & FACL4 activity & Siena \\
\hline & RSK2 activity & Siena \\
\hline \multirow[t]{5}{*}{ Linkage analysis } & Intervals & Laboratory \\
\hline & Xq12-13 & Troina \\
\hline & $\mathrm{Xq} 21-22$ & Roma \\
\hline & $\mathrm{Xq} 22-24$ & Siena \\
\hline & $\mathrm{Xq} 26-28$ & Milano \\
\hline \multirow[t]{2}{*}{ Array-CGH } & Analysis & Laboratory \\
\hline & Whole genome & $\begin{array}{l}\text { Pavia, Siena } \\
\text { Troina }\end{array}$ \\
\hline \multirow{3}{*}{ Other tests } & X chromosome & Siena \\
\hline & Test & Laboratory \\
\hline & $\mathrm{X}$-inactivation & Milano \\
\hline
\end{tabular}

also sporadic cases in which the proband is a male. After the first 2 years, 56 familial, nine likely familial, and 81 sporadic cases have been inserted in the database.

An innovative feature of this biological database is the accuracy of clinical data collection using a combination of descriptive and iconographic tools: text areas, JPEG format photographs, and $20 \mathrm{sec}$ movies. The availability of a detailed description will allow a better definition of the phenotype, since patients with the molecular defect will be identified; this may help researchers in the characterization and definition of new syndromes.

Given that part of the laboratories involved in the project have expertise in lymphoblastoid cell lines culture and DNA storage procedures, a characteristic of the biobank described here is the absence of a main center for the storage of biological samples. All participating laboratories able to establish lymphoblastoid cell lines may propose to store some of the samples. Requirements that a laboratory candidate for biobanking activities must guarantee are those established by the referral guidelines [Godard et al., 2003]. In our opinion these collaborative efforts permit a better use of resources due to a distribution of laboratory costs and operators working time. After the first 2 years of activity, approximately $70 \%$ of biological samples are stored in Siena and $30 \%$ in Rome.

A truly collaborative effort has been made to collect detailed molecular data about each patient. For this aim, mutation analysis is performed on all patients for all genes in study. Linkage analysis for families with at least three affected individuals 
in two generations and exclusion/compatibility mapping for smaller families are performed. Finally, we perform array-based comparative genomic hybridisation $(\mathrm{CGH})$ in all enrolled patients to investigate segmental submicroscopic aneuploidies.

The XLMR bank is an ongoing dynamic database. New clinical centers and laboratories can join the Network at any time just by requesting an appropriate level password from the bank curator. This flexibility allows to meet the demands deriving from scientific knowledge.

Each newly identified mental retardation gene could be added to the list of the molecular tests performed, as soon as a laboratory becomes available. At the same time, the members of the Network may decide to stop the analysis of genes for which the testing is of unproven utility according to the scientific community.

A clinical center may ask for the appropriate level password just to include a single family or a single patient in the database. This allows a more comprehensive collection of interesting cases that have been seen also in small clinical centers spread overall Italy.

To our knowledge the XLMR bank is a unique resource that combines molecular data with accurate clinical information. The XLMR bank with the biobank service and the collection of detailed clinical information represents an important tool for researchers in the field of mental retardation. The availability for the scientific community of biological samples and clinical information from our bank may help in the identification of new mental retardation causative genes and in the delineation of new syndromes.

\section{ACKNOWLEDGMENTS}

The XLMR bank is partially supported by grants from the Telethon Foundation (GTF02006; GTF05005 to A.R.) and the Piertranco and Luisa Mariani Foundation.

\section{APPENDIX: A}

The XLMR Italian Network members are: Amoroso A. (Università di Torino, Italy), Bassi M.T. (IRCSS E. Medea, Bosisio Parini, Italy), Battaglia A. (Istituto Stella Maris, Pisa, Italy), Bedeschi M.F. (Clinica Mangiagalli, Milano, Italy), Bigoni S. (Università di Ferrara, Italy), Borgatti R. (IRCSS E. Medea, Bosisio Parini, Italy), Carnevale F. (P.O. Giovanni XXIII, Bari, Italy), Caruso U. (Istituto Gaslini, Genova, Italy), Cavalli P. (Istituti Ospitalieri, Cremona, Italy), Chiurazzi P. (UCSC, Roma, Italy), D'Alessandro E. (Università dell'Aquila, Italy), D'Avanzo G. (A.O. S.G. Moscati, Avellino, Italy), De Marchi M. (Università di Torino, Italy), Di Rocco M. (Istituto Gaslini, Genova, Italy), Faravelli F. (Ospedale Galliera, Genova, Italy), Ferrero G. (Ospedale Regina Margherita, Torino, Italy), Fichera M. (IRCCS Oasi, Troina, Italy), Fischetto R. (P.O. Giovanni XXIII, Bari, Italy), Galasso C. (Università Tor Vergata, Roma, Italy), Garavelli L.
(Arcispedale S. Maria Nuova, Reggio Emilia, Italy), Guerrini R. (Istituto Stella Maris, Pisa, Italy), Hladnik U. (IRCCS Burlo Garofolo, Trieste, Italy), La Marca G. (Ospedale Meyer, Firenze, Italy), Lerone M. (Istituto Gaslini, Genova, Italy), Marchina E. (Università di Brescia, Italy), Mazzanti L. (Ospedale S. Orsola, Bologna, Italy), Memo L. (USSL9, Treviso, Italy), Micheli V. (Università di Siena, Italy), Moro F. (Istituto Stella Maris, Pisa, Italy), Murgia A. (Università di Padova, Italy), Neri G. (UCSC, Roma, Italy), Pantaleoni C. (Istituto Besta, Milano), Pergola E.M. (USL RME, Roma, Italy), Priolo M. (Ospedali Riuniti, Reggio Calabria, Italy), Rinaldi M.M. (Ospedale Cardarelli, Padova, Italy), Rinaldi R. (Ospedale S. Camillo, Roma, Italy), Romano C. (IRCCS Oasi, Troina, Italy), Russo S. (Istituto Auxologico Italiano, Milano), Scarano G. (A.O.R.N., G. Rummo, Benevento, Italy), Selicorni A. (Clinica Pediatrica De Marchi, Milano, Italy), Sestini S. (Università di Siena, Italy), Stangoni G. (Ospedale di Assisi, Assisi, Italy), Strisciuglio P. (Ospedale Pugliese, Catanzaro, Italy), Tenconi R. (Università di Padova, Italy), Toniolo D. (DIBIT, Milano, Italy), Turolla L. (USSL, Treviso, Italy), Verri A. (IRRCS C. Mondino, Pavia, Italy), Zammarchi E. (Ospedale Meyer, Firenze, Italy), Zelante L. (IRCSS S. Giovanni Rotondo, Italy), and Zuffardi $\mathrm{O}$ (Università di Pavia, Italy).

\section{REFERENCES}

Baird PA, Sadovnick AD. 1985. Mental retardation in over half-a-million consecutive livebirths: an epidemiological study. Am J Ment Defic 89: 323-330.

Bauters M, Van Esch H, Marynen P, Froyen G. 2005. X chromosome array-CGH for the identification of novel X-linked mental retardation genes. Eur J Med Genet 48:263-275.

Chelly J, Mandel J. 2001. Monogenic causes of X-linked mental retardation. Nat Rev Genet 2:669-680.

Dagna Bricarelli F, Baldo C, Filocamo M, Monaco L. 2003. Guidelines for genetic biobanks. Analysis 5-6:1-20.

Gecz J. 2004. The molecular basis of intellectual disability: novel genes with naturally occurring mutations causing altered gene expression in the brain. Front Biosci 9:1-7.

Godard B, Schmidtke J, Cassiman J, Ayme S. 2003. Data storage and DNA banking for biomedical research: informed consent, confidentiality, quality issues, ownership, return of benefits. A professional perspective. Eur J Hum Genet 11(Suppl 2):S88-S122.

McLaren J, Bryson SE. 1987. Review of recent epidemiological studies of mental retardation: prevalence, associated disorders, and etiology. Am J Ment Retard 92:243-254.

Mulley JC, Kerr B, Stevenson R, Lubs H. 1992. Nomenclature guidelines for X-linked mental retardation. Am J Med Genet 43:383-391.

Neri G, Chiurazzi P. 1999. X-linked mental retardation. Adv Genet 41: 55-94.

Renieri A, Pescucci C, Longo I, Ariani F, Mari F, Meloni I. 2005. Non-syndromic X-linked mental retardation: from a molecular to a clinical point of view. J Cell Physiol 204:8-20. 